\title{
Squamous differentiation portends poor prognosis in low and intermediate-risk endometrioid endometrial cancer
}

hospital de amor

Diocésio A.P. Andrade ${ }^{a}$, Vinicius D. Silva ${ }^{b}$, Graziela M. Matsushitab, Marcos A. Limac, Marcelo A. Vieirad, Carlos E. M. C. Andrade ${ }^{d}$, Ronaldo L. Schmidt ${ }^{d}$, Rui M. Reis ${ }^{e}$, Ricardo dos Reis ${ }^{d}$.

aInORP ONCOCLÍNICAS Group (Oncology Institute of Ribeirão Preto). ${ }^{b}$ Department of Pathology, Barretos Cancer Hospital. 'Epidemiology and Biostatistics, Barretos Cancer Hospital. dDepartment of Gynecologic Oncology, Barretos Cancer Hospital. eMolecular Oncology Research Center, Barretos Cancer Hospital.

\section{OBJECTIVE}

The aim of this study was to investigate other clinical-pathological factors that might influence the recurrence rates of patients diagnosed with low and intermediate-risk endometrioid endometrial cancer.

\section{METHODS}

A case-control study from a cohort retrospective of 196 patients diagnosed with low and intermediate-risk endometrioid endometrial cancer at a single institution between 2009 and 2014 was conducted. Medical records were reviewed to compare clinical (race, smoking, menopause age, body mass index) and pathological (histological characteristics (endometrioid vs endometrioid with squamous differentiation), tumor differentiation grade, tumor location, endocervical invasion, LVSI) features of patients with recurrence (case) and without recurrence (control) of disease. Three controls for each case were matched for age and staging.

\section{RESULTS}

Twenty-one patients with recurrence were found (10.7\%), of which 14 were stage IA, and 7 were stage IB. In accordance, 63 patients without recurrence were selected as controls. There were no significant differences in any clinical characteristics between cases and controls (Table 1). Among pathological variables, presence of squamous differentiation (28.6\% vs. $4.8 \%, p=0.007)$, tumor differentiation grade 2 or $3(57.1 \%$ vs. $30.2 \%, p=0.037)$ and presence of endocervical invasion $(28.6 \%$ vs. $12.7 \%, p=0.103)$ were associated with disease recurrence on univariate analysis (Table 1$)$. On multivariable analysis, only squamous differentiation was a significant risk factor for recurrence $(p=0.031)($ Table 2).

Table 1. Univariate analysis of predictive recurrence for low and intermediate-risk endometrioid endometrial cancer

\begin{tabular}{|c|c|c|c|c|}
\hline & & Case $(n=21)$ & Control (n=63) & $P$-value \\
\hline Age $\left(\right.$ median) ${ }^{\mathrm{a}}$ & & $64(46-77)$ & $64(46-78)$ & 0.873 \\
\hline \multirow{2}{*}{ FIGO staging $(\%)^{b}$} & IA & $14(66.7)$ & 42. 66.7$)$ & $>0.99$ \\
\hline & IB & $7(33.3)$ & $21(33.3)$ & \\
\hline \multirow{2}{*}{ ECOG Performance Status (\%) ${ }^{b}$} & $0-1$ & $20(95.2)$ & $61(96.8)$ & $>0.99$ \\
\hline & 2 & $1(4.8)$ & $2(3.2)$ & \\
\hline \multirow{2}{*}{ Race/Ethnicity (\%) ${ }^{b}$} & White & $18(85.7)$ & $45(71.4)$ & 0.251 \\
\hline & Non-white & $3(14.3)$ & $18(28.6)$ & \\
\hline \multirow{2}{*}{$\mathrm{BMI}(\text { median})^{\mathrm{a}}$} & & 31.64 & 32.65 & 0.339 \\
\hline & & $(19.78-48.62)$ & $(21.93-52.71)$ & \\
\hline \multirow{2}{*}{ Smoking historyb } & Yes & $2(9.5)$ & $4(6.3)$ & 0.637 \\
\hline & No & $19(90.5)$ & $59(93.7)$ & \\
\hline \multirow{2}{*}{ Menopause $(\%)^{b}$} & Yes & 21 & $56(88.8)$ & 0.184 \\
\hline & No & 0 & $7(11.2)$ & \\
\hline \multicolumn{2}{|l|}{ Number of pregnancies (median)a } & $4(1-7)$ & $4(1-20)$ & 0.725 \\
\hline \multirow{2}{*}{ Tumor differentiation grade ${ }^{b}$} & Grade 1 & $9(42.9)$ & $44(69.8)$ & 0.037 \\
\hline & Grade 2 or 3 & $12(57.1)$ & $19(30.2)$ & \\
\hline \multirow[b]{2}{*}{ Histological subtype $(\%)^{b}$} & Endometrioid & $15(71.4)$ & $60(95.2)$ & 0.007 \\
\hline & $\begin{array}{c}\text { Endometrioid with squamous } \\
\text { differentiation }\end{array}$ & $6(28.6)$ & $3(4.8)$ & \\
\hline \multirow{2}{*}{ Tumor size $(\text { median }-\mathrm{cm})^{\mathrm{a}}$} & & 4.0 & 4.0 & 0.597 \\
\hline & & $(16.0-115.0)$ & $(1.0-105.0)$ & \\
\hline \multirow{2}{*}{ Tumor localization ${ }^{\mathrm{b}}$} & Uterine corpus & $14(66.7)$ & $47(74.6)$ & 0.574 \\
\hline & Lower uterine segment & $7(33.3)$ & $16(25.4)$ & \\
\hline \multirow{2}{*}{ Endocervical invasion (\%) ${ }^{\mathrm{b}}$} & Yes & $6(28.6)$ & $8(12.7)$ & 0.103 \\
\hline & No & $15(71.4)$ & 55 (87.3) & \\
\hline \multirow{2}{*}{ LVSI (\%) } & Yes & $5(23.8)$ & $9(14.3)$ & 0.324 \\
\hline & No & $16(76.2)$ & $54(85.7)$ & \\
\hline
\end{tabular}

Table 2. Multivariate analysis of predictive recurrence for low and intermediate-risk endometrioid endometrial cancer

\begin{tabular}{|c|c|c|c|}
\hline & & Odds Ratio (IC - 95\%) & $P$-value \\
\hline Tumour differentiation grade & $\begin{array}{c}\text { Grade } 1 \\
\text { Grade } 2 \text { or } 3\end{array}$ & $\begin{array}{c}1 \\
2.66(0.89-7.96)\end{array}$ & 0.080 \\
\hline Tumour type & $\begin{array}{l}\text { Endometrioid } \\
\text { Endometrioid with squamous differentiation }\end{array}$ & $\begin{array}{c}1 \\
5.65(1.17-27.17)\end{array}$ & 0.031 \\
\hline Endocervical invasion & $\begin{array}{l}\text { No } \\
\text { Yes }\end{array}$ & $\begin{array}{c}1 \\
2.55(0.67-9.66)\end{array}$ & 0.168 \\
\hline
\end{tabular}

\section{CONCLUSION}

This case-control study provides evidence that squamous differentiation in low and intermediate risk endometrial cancer had a 5.6-fold increased risk for recurrence. 